\title{
The Influence of Motivation on Ego Depletion in College Athletes
}

\author{
Lihong Sun \\ Dept. of P.E., The No.2 Hubei Institute of Education, Wuhan Hubei 430205, China \\ renwenjijin@126.com
}

\begin{abstract}
The negative effects of ego-depletion may cause the reduction of effort and performance of consecutive task, it's important for college student to develop coping method to counteract ego-depletion and minimize the negative effects. Present research, based on former research, investigate the influence of motivation on ego-depletion via Stroop task, and put forward that internal motivation and external incentive are effective method to cope the unwanted effect of ego-depletion. The results of present research provides another empirical support for ego-depletion, and suggests that internal stimulus and external reward are effective methods to compensate ego-depletion, and inner learning has no compensatory effect on ego- depletion; External reward and external honor both are effective methods to compensate egodepletion, meanwhile the effect is equal. Results support the reserved energy hypothesis for limited resource theory of self-control.
\end{abstract}

Keywords-motivation acting; ego-depletion; internal motivation; external reward

\section{RAISE QUESTIONS}

The ability of self-regulation is the key factor to individual success. From daily eating to dealing with others, life is full of temptation and conflict. Self-regulations are needed. But Self-regulation is a limited resource, once it is used, depletion occurs.Ego-depletion is refered to such an phenomenon: our ability of self-control decreased temporarily once we try to control ourselves, and the decrease also have a bad effect on the subsequent operations.There are much more temptations in sports field, money, fame and sports cars for instance. Athletes need good self-control capacity, especially in time of the key moment of a competition, occupying high level of concentration and self-control. If ego-depletion appears at that time, it may end up with a total failure. There is no shortage of examples of failures. Therefore, exploring athlete's self-depletion problem, especially the strategies for ego-depletion, has important theoretical value and practical significance.

Internal motivation is not only the psychological motive and internal driving force to inspire and sustain the activities of individual,but an indispensable factor in the process of Self-regulation. When the individual is in the state of ego-depletion, internal motivation can play an important role of self resource alternative to some degree.But for athletes, whether internal motivation can successfully deal with ego-depletion, enhance self-control and contribute to a good athletic performance in daily training or competitions are explored in this paper.

\section{EXPERIMENT INTERNAL LEARNING AND INTERNAL STIMULATION FOR EGO-DEPLETION COMPENSATION}

\section{A. Purpose of the experiment}

The purpose of the experiment is to test whether the different dimensions of internal motivation can compensate ego-depletion.

The research hypothesis: internal stimulation and internal learning compensation can improve performance of the Stroop operation after ego-depletion.

\section{B. Participants}

Convenient sampling, we selected 60 sports specialized students(36 males, 24 females),mean of age $M=18.521$, and $\mathrm{SD}=2.034$. They are all right handed and in normal vision.We numbered every participant and divided them into 6 groups in complete random through Excel.All participants finished their experiments in Laboratory and every time there were six people at the same time to complete the experiment.

\section{Experimental Materials and equipment}

We used Interference game video, Self-control manipulation Checklist, Brief Mood Introspection Scale, BMIS and Stroop task.

\section{Experimental Design}

We used $3 \times 2$ between groups design. Independent variables are internal motivation(Learning, stimulation, amotivation), ego-depletion(one of two groups are none ego-depletion). The dependent variables are correct number of color word match words and none color word match words and reaction time measured by Stroop task.

\section{E. Procedure}

These are double-blind experiments, and we trained 6 main trial to cover all experiments process. There were seven steps to complete the experiments.

\section{F. Results}

1) Self-manipulation check. This study used SPSS16.0 analysis software to process data. The independent variable is the type of ego-depletion and the dependent variables are 
the difficulty, level of effort and mood score. Data were analyzed by ANOVA. Mean and standard deviation of every group are shown in Table 1

TABLE 1 THE MEAN AND STANDARD DEVIATION

\begin{tabular}{|c|c|c|c|c|c|c|c|c|c|c|c|c|}
\hline \multirow{3}{*}{ Dependent variables } & \multirow{2}{*}{\multicolumn{2}{|c|}{ stimulation }} & \multicolumn{4}{|c|}{ ego-depletion group } & \multicolumn{4}{|c|}{ None ego-depletion group } & & \\
\hline & & & \multicolumn{2}{|c|}{ Learning } & \multicolumn{2}{|c|}{ amotivation } & \multicolumn{2}{|c|}{ stimulation } & \multicolumn{2}{|c|}{ Learning } & \multicolumn{2}{|c|}{ amotivation } \\
\hline & $M$ & $S D$ & $M$ & $S D$ & $M$ & $S D$ & $M$ & $S D$ & $M$ & $S D$ & $M$ & $S D$ \\
\hline The difficulty & 3.300 & 1.636 & 3.800 & 1.229 & 3.500 & 1.650 & 3.300 & 1.059 & 2.400 & 1.075 & 2.500 & 1.179 \\
\hline Level of effort & 3.400 & 1.252 & 3.000 & 0.816 & 3.200 & 0.919 & 2.400 & 0.843 & 2.200 & 0.632 & 3.000 & 1.155 \\
\hline Mood score & 43.600 & 4.926 & 42.400 & 5.661 & 41.400 & 5.481 & 43.000 & 6.944 & 43.000 & 3.859 & 46.700 & 7.379 \\
\hline
\end{tabular}

The type of ego-depletion have a significant main effect on the difficulty, $F(1,54)=5.445, p=.023, \eta^{2}=.092$. That is to say, ego-depletion group's ( $M=3.530, S D=1.479$ ) evaluation of the subjective difficulty on the task is higher significantly than none ego-depletion group's ( $M=3.130, S D=1.371$ ) ; the main effect of the type of Internal motivation on the difficulty was not significant, $F(2,54)=0.265, p=.768$, $\eta^{2}=.010$.

The type of ego-depletion have a significant main effect on the level of effort, $F(1,54)=3.060, p=.046, \eta^{2}=.054$. That is to say, ego-depletion group ( $M=3.263, S D=0.977$ ) thought they should pay more effort to complete the task than none ego-depletion group $(M=2.555, S D=0.833)$, the main effect of the type of Internal motivation on the level of effort was not significant, $F(2,54)=2.010, p=.144$, $\eta^{2}=.069$.

The type of ego-depletion have a significant main effect on mood score, $F(1,54)=1.377, p=.246, \eta^{2}=.025$. That is to say, there is no differences between ego-depletion group ( $M=42.944, S D=1.901)$ and none ego-depletion group ( $M=44.363, S D=1.577$ ) on mood score.

In conclusion, the subjective difficulty and level of effort was significant, showing that it is effective to operate the ego-depletion task. And mood scores were not significant, showing that the mood have no effect on ego-depletion.

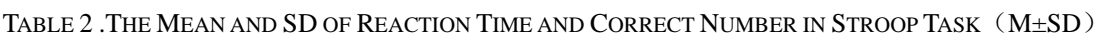

\section{2)Stroop task performance}

Independent variables are internal motivation and ego-depletion. The dependent variables are correct number and reaction time of color word match words and none color word match words measured by Stroop task. We used SPSS16.0 analysis software to process data. The mean and SD of reaction time and correct number of color word match words and none color word match words can be seen in Table 2.

In multivariate ANOVA, the dependent variables test showed that there is a significant difference between different ego-depletion groups in Stroop task, $F(4$, $51)=6.637, p=.000, \eta^{2}=.342$; different dimensions of internal motivation group had no significant difference in Stroop task, $F(8,104)=1.349, p=.228, \eta^{2}=.094$; the interaction between type of ego-depletion and internal motivation is significantly different in Stroop task, $F(8,104)$ $=3.073, p=.004, \eta^{2}=.191$; and multivariate Analysis of Variance overall test results showed that type of ego-depletion and the interaction between type of ego-depletion and internal motivation had an significant effect on Stroop task results. It is essential to have a further analysis, and the results were seen in Table 3.

\begin{tabular}{|c|c|c|c|c|c|}
\hline \multirow{3}{*}{ ego-depletion } & \multirow{3}{*}{ internal motivation } & \multicolumn{2}{|c|}{ correct number } & \multicolumn{2}{|c|}{ reaction time } \\
\hline & & match condition & none match condition & match & none match condition \\
\hline & & & & condition & \\
\hline \multirow{3}{*}{ ego-depletion } & stimulation & $68.600 \pm 3.314$ & $34.700 \pm 1.252$ & $683.540 \pm 71.035$ & $616.130 \pm 32.103$ \\
\hline & Learning & $67.100 \pm 2.923$ & $34.500 \pm 1.269$ & $698.130 \pm 71.179$ & $689.130 \pm 65.412$ \\
\hline & amotivation & $67.300 \pm 3.093$ & $33.300 \pm 2.494$ & $734.820 \pm 40.640$ & $736.210 \pm 90.106$ \\
\hline \multirow{3}{*}{ none ego-depletion } & stimulation & $65.700 \pm 5.417$ & $33.600 \pm 3.273$ & $694.530 \pm 255.763$ & $781.100 \pm 102.116$ \\
\hline & Learning & $65.100 \pm 3.348$ & $32.700 \pm 3.713$ & $755.20 \pm 61.861$ & $756.170 \pm 97.810$ \\
\hline & amotivation & $61.400 \pm 5.892$ & $32.200 \pm 3.584$ & $591.356 \pm 60.130$ & $650.500 \pm 128.004$ \\
\hline
\end{tabular}


TABLE 3 CORRECt Number AND REACTION TIME IN STROOP TASK ANALYSED By ANOVA

\begin{tabular}{|c|c|c|c|c|}
\hline Sources of variation & dependent variables & $d f_{2}$ & $F$ & $p$ \\
\hline & Correct number & & & . \\
\hline & on match condition & 54 & 11.302 & 601 \\
\hline & reaction time & & & \\
\hline \multirow[t]{7}{*}{ ego-depletion } & on match condition & 54 & 0.196 & .660 \\
\hline & Correct number & & & \\
\hline & on none match condition1 & 54 & 2.929 & .093 \\
\hline & reaction time & & & \\
\hline & on none match condition 1 & 54 & 4.298 & $.043^{*}$ \\
\hline & Correct number & & & \\
\hline & on match condition & 54 & 1.212 & .306 \\
\hline ego-depletion & reaction time & & & \\
\hline$*$ & on match condition & 54 & 0.596 & .555 \\
\hline \multirow[t]{4}{*}{ internal motivation } & Correct number & & & \\
\hline & on none match condition2 & 54 & 0.169 & .845 \\
\hline & reaction time & & & \\
\hline & on none match condition 2 & 54 & 9.613 & $.000^{* *}$ \\
\hline
\end{tabular}

\section{DISCUSSION AND ANALYSIS}

condition, $F(1,54)=4.298, p=.043, \eta^{2}=.074$, and the interaction between the type of ego-depletion and internal motivation on reaction time on none match condition is very significant, $F(2,54)=9.613, p=.000, \eta^{2}=.263$. Simple effect test showed that, on condition of ego-depletion, the differences between stimulation group and amotivation group are very significant on reaction time on none match condition, $p=.000$. Thus, the reaction time of stimulation group on none match condition $(M=616.130, S D=32.103)$ was significantly shorter than that of amotivation group ( $M=736.210, S D=90.106$ ) . There is no difference between learning group and amotivation group on reaction time on none match condition, $p=.127$; and the reaction time of stimulation group on none match condition $(M=616.130$, $S D=32.103$ ) was significantly shorter than that of learning group ( $M=689.130, S D=65.412)$. On condition of none ego-depletion, main effect of Internal motivation was not significant, $F(8,50)=1.508, p=.178, \eta^{2}=.194$. The results suggested that On condition of ego-depletion, the internal stimulation exert an effect on the reaction time on none match condition for participants, meaning that stimulation have an compensation effect on ego-depletion, but on condition of none ego-depletion, internal motivation have no effect on the reaction time on none color word match

\section{A. The effect of Internal motivation on reaction time and mood}

As can be seen from the above results, The reaction time on none match condition as the dependent variable partially supported the hypothesis: the reaction time of ego-depletion group on none match condition was significantly longer than that of none ego-depletion group, suggesting that ego-depletion task successfully induced participants' ego-depletion. When internal motivation started, the internal stimulation effectively compensate for the ego-depletion. The reaction time of learning group on none match condition was significantly longer than that of internal stimulation group, suggesting that participants in Internal stimulation group have a better performance than others in learning group and amotivation group in the second task, meaning that only internal stimulation can compensate for the ego-depletion.

Mood scale score differences were found, meaning that internal motivations compensation effect for ego-depletion is not by raising emotion.

\section{B. Internal stimulation compensated for ego-depletion}

Previous studies found that positive experience helped to compensated for individual energy which had been lost 
in the initial self-control task. The study also verified this conclusion, internal stimulation is not only a positive experience, but also a kind of self-regulation. Its role is mainly reflected in two aspects. On the one hand, independent regulation of self-control itself faced less conflict, and therefore less self-control energy was lost. On the other hand, Independent demand meets the increased subjective energy, resulting in rise of the subsequent self-control capacity, or played a complementary role in compensating ego-depletion energy. It is very important to people's lives, we need self-control, and self-control is the core functions of the ego. It can initiate, maintain and regulate human behaviors. Failure of self-control is often associated with bad results, such as personal internal conflicts, low achievement, over-eating, response bias, excessive consumption and so on. But ego is a limited resource, self-control will cause consumption, making a lack of available resources for following tasks. And this is why self-control failed. Internal motivation provides a response to the loss of self-control.

\section{CONCLUSION}

This study provides empirical support for ego-depletion theory again, finding that when selecting Stroop task for the operator task, internal stimulation have an compensation effect on ego-depletion, but internal learning doesn't have such an compensation effect.

\section{REFERENCE}

[1] Baumeister, R. F., \& Heatherton, T. E. (1996). Self-regulation failure: An overview. Psychological Inquiry, 7, 1-15.

[2] Baumeister, R. F., Bratslavsky, E., Muraven, M., \& Tice D. M. (1998). Ego-Depletion: Is the Active Self a Limited Resource? Personality Processes and Individual Differences, 74(5), 1252-1256.

[3] Baumeister, R. F., DeWall. N. C., Ciarocco, N., \& Twenge. (2005). Social Exclusion Impairs Self-Regulation. Journal of Personality and Social Psychology, 88(4), 589-604.

[4] Baumeister, R. F. (2002). Ego depletion and self-control failure: An energymodel and the self's executive function. Self and Identity, 1, 129-136.

[5] Baumeister, R. F. (2003). Ego depletion and self-regulation failure: Aresource model of self-control. Alcoholism: Clinical and Experimental Research, 27, 281-284.

[6] DeWall, C. N., Baumeister, R. F., Stillman, T. F. \& Gailliot, M.T. (2007). Violence restrained: Effects of self-regulatory capacity and its depletion on aggressive behavior. Journal of Experimental Social Psychology, 43:62-76.

[7] Gailliot, M. T., \& Baumeister, R.F. Self-regulation and sexual restraint: Dispositionally and temporarily poor self-regulatory abilities contribute to failures at restraining sexual behavior. Personality and Social Psychology Bulletin. 